\title{
Embedded Implementation of YOLO Nano Algorithm for On-Site Recognition of Relay Pressure Board Status
}

\author{
Xinhai $\mathrm{LI}^{1}$, Dehe FAN, Chenxu MENG, Lingcheng ZENG, Tuolai YUAN \\ Zhongshan Power Supply Bureau of Guangdong Power Grid, Zhongshan, Guangdong \\ 528401, China
}

\begin{abstract}
For the relay platen state identification problem, whether it is a camera or a designated track driving robot inspection, will be affected by the glass door reflection, foreign object obstruction, angle blind area and other platen can not be correctly identified, manual inspection is inevitable. In addition, the current platen image recognition requires a huge background structure, and the inspectors cannot immediately obtain the verification information at the operation site, which greatly affects the work efficiency. In this paper, in response to the above problems, an embedded-based handheld terminal solution is proposed, which uses a miniature intelligent HD camera to achieve multi-angle photography of the pressure plate screen cabinet, effectively solving the problem of obstructing photography due to glass door reflection, foreign object obstruction, angle, etc. In addition, this paper proposes a relay platen status field identification method based on embedded and YOLO Nano algorithm, and selects a high-performance computing core and a special vision recognition module as the hardware platform to avoid a huge background architecture and realize the operation field identification and verification. The test results show that the system has a $100 \%$ accuracy rate of relay platen status identification, which effectively solves the operation site identification problem of relay platen status and has strong practicality and applicability.
\end{abstract}

Keywords. Relay protection, relay pressure plate status field identification, embedded image recognition system, handheld terminals, YOLO Nano

\section{Introduction}

The pressure plate data in the relay room in the substation is huge, and the traditional pressure plate inspection and verification methods are divided into manual inspection and verification and orbital robot inspection and verification. Due to the huge number of pressure plates, the efficiency of manual checking one by one at the operation site is low; while the designated orbiting robot inspection will be plagued by glass door reflection, foreign object obstruction, angle blindness and other pressure plates cannot be correctly identified; in addition, the current pressure plate image recognition requires a huge background structure, and the inspection personnel cannot immediately

\footnotetext{
${ }^{1}$ Corresponding Author, Xinhai LI, Nr. 5, Xihe East Road, West District, Zhongshan City, Provincie Guangdong; E-mail: zslixinhai@163.com.
} 
obtain the verification information at the operation site, which greatly affects the efficiency.[1-3]

In this paper, to address the above problems, an embedded-based handheld terminal solution is proposed, which adopts Kodak PixPro SL10 miniature intelligent HD camera to realize multi-angle photography of the pressure plate screen cabinet, effectively solving the problems of obstructing photography due to glass door reflection, foreign object obstruction and angle; in addition, this paper proposes an embedded and YOLO Nano algorithm-based relay In addition, this paper proposes an on-site identification method of pressure plate status based on embedded and YOLO Nano algorithm, and selects 6-core Cortex-A series high-performance computing core and Mali-T860, a special visual identification module, as the hardware platform to perform pressure plate verification by reading the table of the scheduling pressure plate mode pre-existing on the terminal, which avoids the huge background architecture and realizes the batch identification and verification of pressure plate status at the operation site, solving the problem of low work efficiency of manual one-by-one verification at the operation site. The problem of low work efficiency is solved.

The method proposed in this paper is used in a $220 \mathrm{kV}$ substation relay room ( 300 screen cabinets, 7088 pressure plates) for on-site verification of relay pressure plate status, with $100 \%$ equipment performance and algorithm efficiency, and 100\% accuracy of pressure plate identification. It can improve the efficiency and accuracy of pressure plate status identification and verification, avoid human errors, and is easy to promote the use.

\section{The Research Idea of this Paper}

In order to realize the on-site identification and verification of the relay platen throwout operation, the overall design scheme of the proposed method includes two parts: the hardware platform of the handheld terminal and the firmware program software. Embedded hardware platform core for image recognition embedded unit consists of image acquisition module, graphics processing module, AI computing module and display module. The operation site platen recognition check flow, as shown in figure 1.

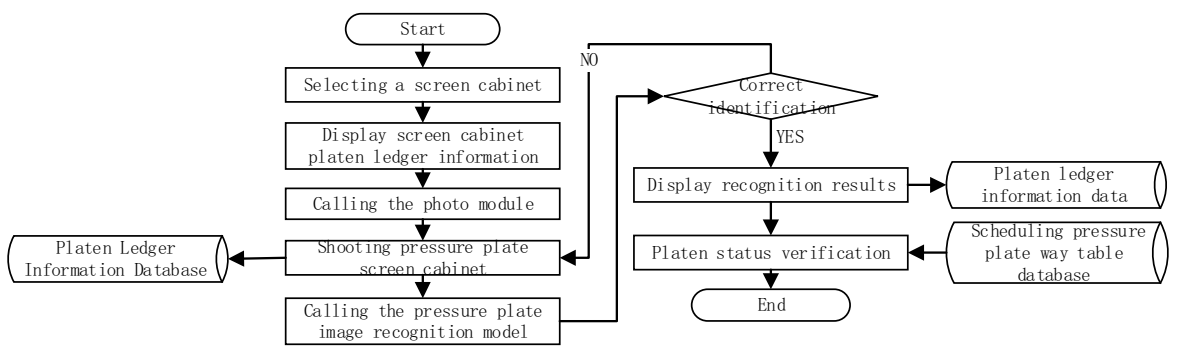

Figure 1. Checking process of platen status identification

Through the image acquisition module, the image of the relay platen is acquired; then the camera lens transmits the image to the handheld terminal for storage through WIFI interface; the graphics processing module and AI computing module process and identify the captured relay platen image based on computer vision and YOLO Nano target identification algorithm; then the identification result and check report are displayed through the display module, and at the same time, the data can be stored 
through WIFI network, USB interface to transfer data to the server for storage. The image acquisition module adopts Kodak PixPro SL10 lens camera to realize detachable lens and high-definition photography. The graphics processing and recognition module adopts ARM Mali-T860 and 6-core Cortex-A series chips to realize the processing, convolution calculation and target recognition of the captured images.

\section{Handheld Terminal Structure for On-site Identification and Verification of Platen Status}

The core of the embedded handheld terminal for the status of the relay platen is an image recognition embedded unit consisting of an image acquisition module, a graphics processing module, an AI calculation module and a display module, as shown in figure 2.

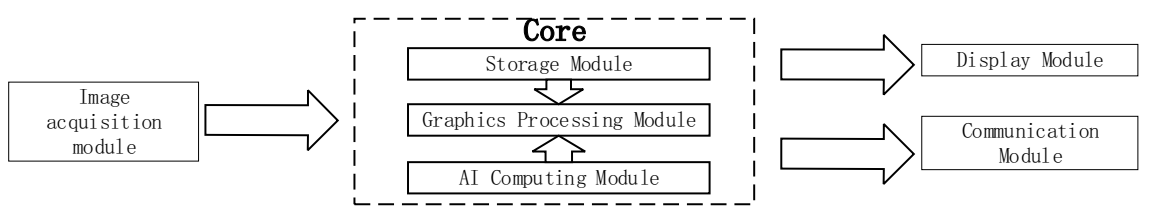

Figure 2. Relay platen status identification handheld terminal hardware structure

\subsection{Image Acquisition Module}

The image capture module uses the Kodak PixPro SL10 lens camera, whose biggest advantages: 1 . can be detached from the handheld terminal host; 2 . the module is a miniature smart camera; 3. can achieve high-definition photography; 4 . can be applied with a variety of authoring-style devices; 5 . easy to carry. The module integrates a 16.35 MP 1/2.3-inch BSI CMOS sensor for high-resolution shooting at up to $6 \mathrm{fps}$, and the sensor's BSI design enhances low-light performance and quality for ISO3200 sensitivity. The module includes a 10x zoomable lens that offers a range of 28 to 280 $\mathrm{mm}$, equivalent to a $35 \mathrm{~mm}$ iso-focal length, covering wide angle to telephoto views. The module features optical image stabilization that minimizes camera shake to deliver sharper photos. The module utilizes built-in Wi-Fi connectivity, plus the ability to pair devices with NFC (Near Field Communication), which can be set up by the core host to control and shoot, and transfer photos to the handheld terminal host via WIFI, the image capture module, as shown in figure 3. 

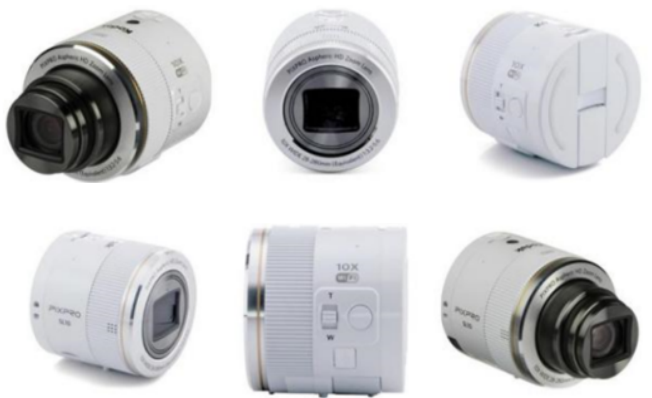

Figure 3. Image acquisition module

\subsection{Storage Module}

Since the photos are taken in high definition, the photo files are relatively large and need to be read quickly. The system described in this paper uses a high-speed embedded multimedia card (eMMC) with a capacity of $128 \mathrm{~GB}$ as the storage medium, which ensures the system operation efficiency and transmission efficiency. Support MicroSD (TF) Card expansion, and also support PCIE interface SSD expansion, to achieve flexible expansion of storage space, to effectively ensure that the system has enough space to store data.

\subsection{Graphics Processing AI Calculation Module}

The graphics processing AI computing module uses a server-grade dual-core CoretexA72 + quad-core Cortex-A53 large and small core architecture, combined with a quadcore ARM Mali-T860 MP4 GPU to achieve image processing, convolution calculation, target recognition and data interaction functions of each module for filming. Mali-T860 adopts Midgard architecture, which is based on 128bit vector, and is 2 4 times faster than 6-core big.LITTLE CPU when applied to image convolution calculation. The adopted 6-core Contex architecture with higher interface standard and faster transmission speed provides faster computing speed and better visual effect for the terminal.

\subsection{Display Module}

The display module adopts high-definition capacitive touch screen as the core part of the touch display module. Users can select and edit the function and attribute information, analyze data information reading, etc. through the touch display. It supports high-performance display interfaces such as HDMI2.0, DP1.2, MIPI-DSI and eDP, and has powerful display expansion capability.

\subsection{Communication Module}

The system communication interfaces include $2.4 \mathrm{G} \mathrm{Hz} / 5 \mathrm{GHz}$ dual-band WiFi, BT4.1 and Gigabit Ethernet and dual USB3.0 interfaces with transmission rate up to 5Gbps. The trained algorithm model can also be downloaded to the handheld terminal via WiFi 
or USB interface, so that the image recognition algorithm on the handheld terminal can be kept up-to-date.

\section{Embedded Software Design for in Situ Identification and Verification of Platen Status}

The system firmware program proposed in this paper includes two parts: the operating system and the pressure plate state identification software. The operating system firmware includes display driver, WiFi driver, Bluetooth driver, USB driver and operating system porting module. The platen status identification software includes platen ledger information management, platen image acquisition, platen status image identification, platen identification verification report and exception information module. The firmware program structure, as shown in figure 4.

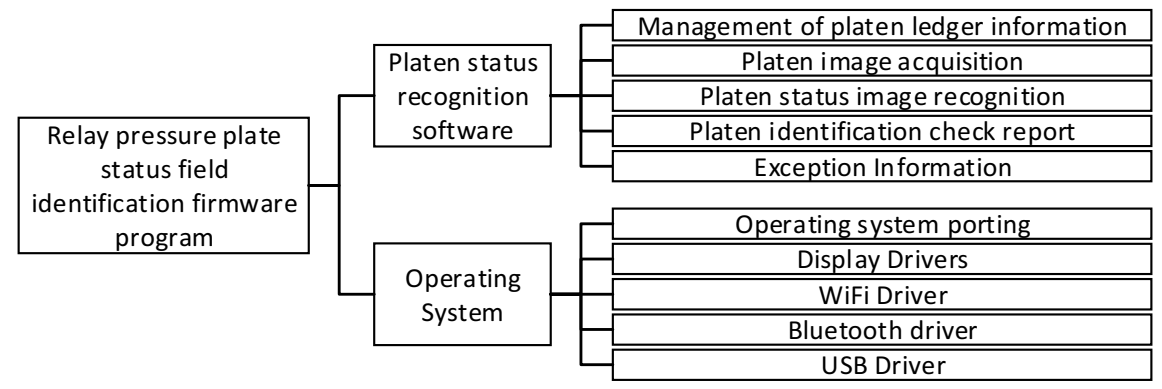

Figure 4. Firmware program structure

\subsection{Embedded Linux OS Firmware}

The embedded operating system is responsible for the allocation of embedded system software and hardware resources, task scheduling and control, and coordination of concurrent activities. The system consists of underlying drivers, system kernel, device drivers, communication protocols, etc. The embedded Linux operating system is characterized by a small kernel, strong specialization, simple system instructions, high real-time capability, and is a multitasking operating system.

System porting needs to be cross-compiled for a specific device in order to be ported to the hardware motherboard. There are three methods of system porting, namely uboot, bootloader and kernel methods. The system porting of the rk3399 hardware platform described in this paper uses the uboot method, compiles the embedded Linux system using the cross-compilation method, and image packages the system, and writes the system image to the rk3399 hardware platform through the uboot porting tool.

The display driver is used to drive the high resolution OLED display display and touch function. WiFi driver and Bluetooth driver are used to drive WiFi and Bluetooth for data transfer communication with the image acquisition module. USB driver is used to drive data transfer communication with third party platforms. 


\subsection{Platen Status Recognition Software}

The platen status recognition software realizes the management of embedded curing and related information based on YOLO Nano algorithm, provides a friendly image interface for user-friendly operation and use, and the software is built and developed using Java platform. The software function modules include: platen image acquisition module, platen ledger information management module, platen status image recognition module, platen recognition check report module and platen recognition check report module.

The pressure plate ledger information management module is used to manage and set the pressure plate ledger information stored on the handheld terminal in the main control room, and can view the original status information of each screen ledger and pressure plate status in the main control room.

Platen image acquisition module, by controlling the image acquisition module to take photos of the platen screen cabinet, to obtain photos of the platen state, and to store and manage the photos.

The platen state image recognition module, by using the TensorRT platform to build the YOLO Nano network model, and training the model, ported to the embedded platform, this module by calling the algorithm to image recognition of the platen photos stored on the device, to get each platen open and closed state information. The platen identification check report module integrates the platen status image identification results to form an easy-to-understand and intuitive platen identification check report. Abnormal information module, displaying the information of the name of the platen that has abnormalities after platen image recognition.

\section{YOLO Nano Algorithm for Relay Platen Status Field Identification}

Due to the limitation of computing resources of embedded devices, in order to maximize the effectiveness of image recognition algorithms. The algorithms deployed on embedded devices are required to have high accuracy, good real-time performance, low development difficulty, small network model, and low memory occupation. The existing target detection networks, such as SSD and Mask-RCNN, are unable to perform their applications on embedded devices due to the limitations of computation and memory of embedded devices.

Using the single-stage target detection network architecture as a prototype, the YOLO Nano network combines prototyping and machine-driven design exploration strategies to create a simple and compact network architecture whose most significant feature is that the network is designed for embedded and edge devices. The network is based on the YOLO family design and inherits the excellent target detection performance of the YOLO algorithm. The network model is 8.3 times smaller than Tiny YOLOv3 and runs 3 3.8 times faster than SSD and RetinaNet, respectively, and supports target detection on embedded GPUs.

The YOLO Nano network is a highly customized network for embedded devices and is created in two steps: 1 . create a prototype network based on the YOLO family of networks; 2. obtain the final network based on the prototype network through a machine-driven exploration strategy. The network is a highly customized network for embedded devices. 
Created based on a prototype network, the prototype network (denoted as $\varphi$ ) consists of a stack of feature representation modules with the same shortcut connections between modules as in YOLOv3. The feature representation module is similar to a pyramid network, allowing the network to represent features in three different dimensions. Efficient and multiscale target detection is achieved by adding a convolutional layer after the feature module to output a 3D tensor of the bounding box, and three different sizes of classification predictions.

The machine-driven exploration phase designs the network, using the prototype network, data and embedded device requirements as a guide to determine the macroarchitecture and micro-architecture of the YOLO Nano by using machine-driven exploration strategies to finally obtain the YOLO Nano network structure. This phase defines the network generator $g$, which generates the network $\left\{N_{s} \mid s \in S\right\}$ of function $u$ by the given seed set $S$. The network satisfies the definition and constraint requirements of the indicator function $l_{r}(\cdot)$, whose expression is shown in Equation (1).

$$
g=\max _{\mathcal{G}} u(g(s)), l_{r}(g(s))=1, \forall s \in S
$$

The iterative algorithm is used to find the approximate solution $\hat{g}$, while $g_{0}$ is used as a guide by $\varphi, u$ and $l_{r}(\cdot)$, which is gradually updated under the constraint of $1 \_r(\cdot)$ to obtain a continuous $g_{k}$, such as $\left(g_{1}, g_{2}, g_{3}, \ldots, g_{k}, \ldots\right)$, and use the final approximation $\hat{g}$ to create the YOLO Nano network.

\section{System Implementation}

\subsection{System Hardware Platform Implementation}

RK3399 of Rexchip Micro is used as the core module with ARM-based Linux operating system to design and develop a handheld terminal for relay platen status identification. The size of the hardware core version of the handheld terminal is $124 \times 93 \mathrm{~mm}$, which meets the design requirement of portable embedded handheld terminal, as shown in figure 5.

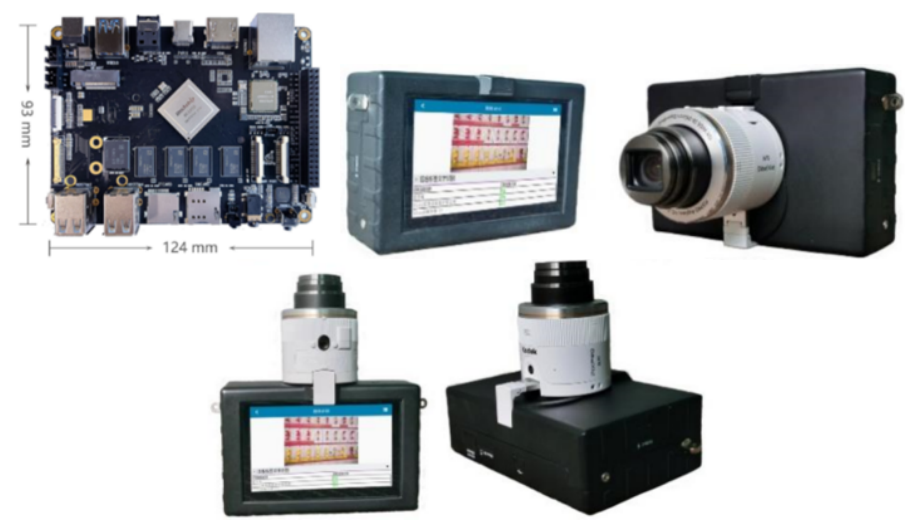

Figure 5. RK3399 Core Motherboard and Implementation of Embedded Handheld Terminal 
The handheld terminal shell is designed as a combination of handheld and neck hanging. The handheld terminal is designed with image acquisition module installation clips on both sides, so that the smart camera lens can be installed on the handheld terminal host through its own clips to realize the diversity of use, and the shell is $3 \mathrm{D}$ printed to realize the effect.

\subsection{System Software and Algorithm Implementation}

The platen state recognition software is built and developed based on Java platform. The YOLO Nano network for embedded devices is built in the host computer using the TensorRT framework, and the network is trained with the labeled data set to obtain the prediction model and parameters, which are embedded in the recognition software. The model and parameters are embedded in the recognition software. The software is installed and deployed in the embedded handheld terminal, and the algorithm model is invoked to recognize the platen image during the usage. Some of the software functions are implemented as shown in figure 6.

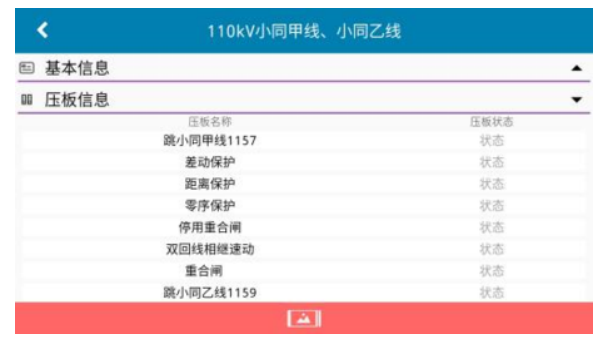

a-Management of platen ledger information

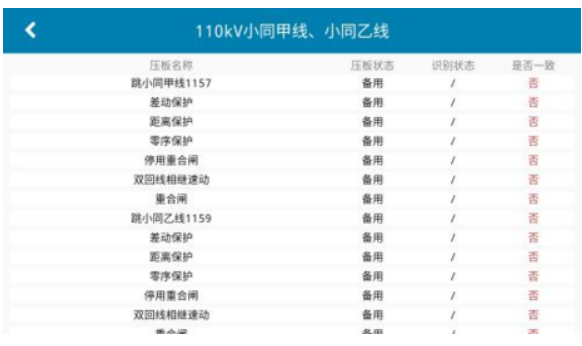

c-Platen image verification report

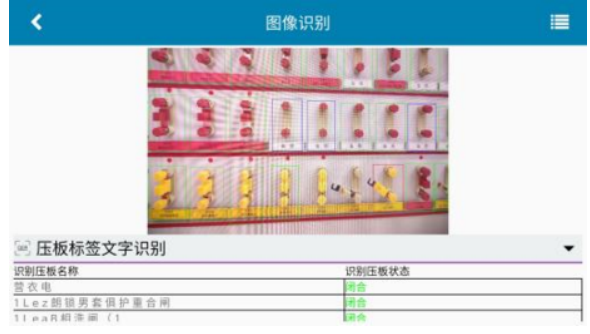

b-Image recognition of platen status

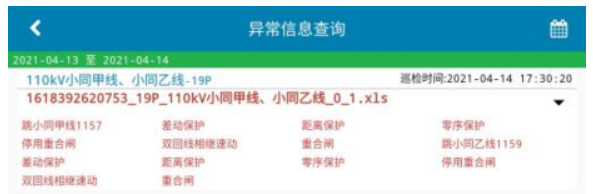

d- Exception information

Figure 6. Platen status recognition software implementation

\section{Analysis of Test Results}

This paper validates the relay protection platen state field recognition system based on embedded and YOLO Nano algorithm, using test image dataset for network model recognition test, the relay protection platen state recognition effect is shown in figure 7. The green box indicates that the pressure plate is in the closed state, red indicates the closed state, and blue is the standby. 


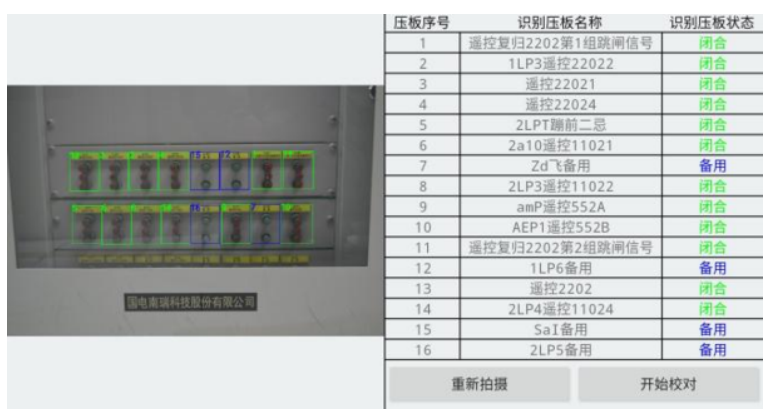

Figure 7. Image recognition effect of platen status

In the main control room of a $220 \mathrm{kV}$ substation, the system proposed in this paper was used to conduct relay pressure plate image identification and verification tests at the operation site, and the test results are shown in table 1 below.

Table 1. Handheld terminal platen image recognition test analysis table

\begin{tabular}{cccc}
\hline Num & Number of plates & Number of correct & Correct recognition rate \\
\hline 1 & 200 & 200 & $100 \%$ \\
2 & 400 & 400 & $100 \%$ \\
3 & 600 & 600 & $100 \%$
\end{tabular}

From the analysis of the experimental results, it can be obtained that the accuracy of platen recognition is $100 \%$ when the YOLO Nano algorithm is used in the embedded handheld terminal for photo recognition of platen status.

\section{Conclusion}

The present relay platen verification has the problems of perceived large influencing factors, difficulty in promoting the verification system and low accuracy of image recognition. In this paper, the identification and verification of the field relay platen status is realized by developing an embedded handheld terminal and curing the YOLO Nano network model in the handheld terminal.(1) The embedded handheld terminal was developed to solve the problem of the convenience of verifying the status of the platen on site. (2) It solves the problem that the current substation image identification application must use the background server to identify, and the site verifier can obtain the pressure plate identification and verification results in time by using the handheld terminal to take pictures of the pressure plate screen cabinet.(3) Innovative use of an embedded device with neural network acceleration function to solidify the YOLO Nano model and apply it to relay pressure plate status verification in the field.(4) After the application of the experimental summary of the proposed method for relay platen status identification accuracy of more than $100 \%$, can effectively solve the problem of low efficiency of manual verification platen status, with strong practical and promotional value. 


\section{References}

[1] Zhou K, Yang Q W, Wang Y Y et al 2021 An improved SSD algorithm based on pressure plate status recognition Electrical Measurement \& Instrumentation, 2021, 58(1): 69-76.

[2] Wang W, Zhang Y L, Zhai D H et al Intelligent identification of substation platen state based on OpenCV + SSD deep learning model Electrical Measurement \& Instrumentation, 2020, 1-10.

[3] Pan C C, Lu Q, Hu L K et al Platen location and status recognition based on image processing and morphological features analysis Electrical Measurement \& Instrumentation, 2020, 1-8. 